\title{
Optical binding of nanowires
}

\author{
Stephen H. Simpson, ${ }^{*} \dagger$ Pavel Zemánek, ${ }^{\dagger}$ Onofrio M. Maragò, ${ }^{\ddagger}$ Philip H. Jones, $₫$ \\ and Simon Hanna ${ }^{\S}$ \\ Institute of Scientific Instruments of the CAS, Kràlovopolskà 147, 61264 Brno, Czech \\ Republic, CNR-IPCF, Istituto per i Processi Chimico-Fisici, Consiglio Nazionale delle \\ Ricerche, Viale F. Stagno D'Alcontres 37, I-98158, Messina, Italy, Department of Physics \\ and Astronomy, University College London, London WC1E 6BT, UK, and H.H. Wills \\ Physics Laboratory, University of Bristol, Tyndall Avenue, Bristol, BS8 1TL, UK \\ E-mail: simpson@isibrno.cz
}

\begin{abstract}
Multiple scattering of light induces structured interactions, or optical binding forces, between collections of small particles. This has been extensively studied in the case of micro-spheres. However, binding forces are strongly shape dependent: here, we turn our attention to dielectric nanowires. Using a novel numerical model we uncover rich behaviour. The extreme geometry of the nanowires produces a sequence of stationary and dynamic states. In linearly polarized light, thermally stable ladder-like structures emerge. Lower symmetry, sagittate arrangements can also arise, whose configurational asymmetry unbalances the optical forces leading to non-conservative, translational motion. Finally, the addition of circular polarization drives a variety of coordinated rotational states whose dynamics expose fundamental properties of optical spin. These

${ }^{*}$ To whom correspondence should be addressed

†'Institute of Scientific Instruments of the CAS, Kràlovopolskà 147, 61264 Brno, Czech Republic

${ }^{\ddagger}$ CNR-IPCF, Istituto per i Processi Chimico-Fisici, Consiglio Nazionale delle Ricerche, Viale F. Stagno D'Alcontres 37, I-98158, Messina, Italy

"Department of Physics and Astronomy, University College London, London WC1E 6BT, UK

${ }^{\S}$ H.H. Wills Physics Laboratory, University of Bristol, Tyndall Avenue, Bristol, BS8 1TL, UK
\end{abstract}


results suggest that optical binding can provide an increased level of control over the positions and motions of nanoparticles, opening new possibilities for driven self organisation and heralding a new field of self-assembling optically-driven micromachines.

\section{Keywords}

Optical binding, nanowires, Brownian motion, self organisation, non-equilibrium thermodynamics, non-equilibrium steady state, spin-orbit coupling, emergent phenomena

The physical properties of nanostructures are strongly influenced by their size and shape. Their arrangement into hierarchical meso-structures, or meta-materials, represents a major theme in materials science. ${ }^{1-4}$ One way to impose ordering on a collection of nano-structures is through optical binding. Optical binding between spherical micro-particles was first explored over twenty five years ago ${ }^{5}$ and has now been applied by numerous researchers. ${ }^{6-13}$ Recently, light mediated self-assembly of more generally shaped nanoparticles has also received attention. ${ }^{2,14-18}$ Multiple scattering of light produces finely textured force-fields whose structure depends both on the shape of the particles and the properties of the incident field. The result is a broad range of organisational possibilities. The formation and coherence of such assemblies result from the interplay between the systematic optical and hydrodynamic forces, together with stochastic thermal fluctuations; a realistic model must include all of these.

For spherical particles, analogies have been drawn between optical interaction forces and the potential-derived forces operating between atoms. ${ }^{2,19}$ The arrangement of optically bound spheres has been likened to a form of nanoscale chemistry, such principles being applied in the formation of nanoparticle lattices, ${ }^{2}$ and the patterning of substrates. ${ }^{20}$ However, while the molecular analogy is appealing, optical interaction forces actually arise from a continuous momentum exchange with an external, momentum bearing field. Unlike their atomic analogues, optically organized structures derive their structural coherence from intrinsically non-conservative forces: appearance notwithstanding, they are not at thermal equilibrium. A 
recent study highlights this: the optical binding forces between two differently-sized spheres are not balanced, with the result that the pair will experience an overall force resulting in motion of the centre of mass. ${ }^{21}$ This demonstrates an important difference between optical binding and chemistry: optical fields can lead to non-equilibrium steady-state structures that move spontaneously. As we will show, non-conservative, non-equilibrium effects become increasingly conspicuous with reducing particle symmetry. Such systems can sustain a broad spectrum of stationary and dynamic states suitable, not only, for arranging and organising nano-structures, but also for driving spontaneous, coordinated motions. Such behaviour is, in a sense, life-like, having the potential for performing detailed tasks at the nano scale.

To investigate these possibilities we use a novel Brownian dynamics approach, based on that of Ermack and McCammon, ${ }^{22}$ that includes shape effects as well as appropriately coupled optical and hydrodynamic interactions and thermal fluctuations. The method is described in detail in the Supplementary Information. The approach relies on representing each nanostructure by a small number of rigidly connected beads, which are used in both the hydrodynamic and optical calculations. Constraint forces which act to preserve the relative positions of the beads are taken into account when updating the particles positions and orientations.

The inclusion of shape effects, and the resulting rotational degrees of freedom, profoundly alter the optical interaction, leading to qualitatively new behaviour, ${ }^{16-18,23,24}$ which we will discuss here in the context of nanowires. In this case, in particular, the interaction forces become anisotropic and the incident polarization plays an important role.

We will consider high index, high aspect ratio nanowires in counter-propagating light beams. This system has been chosen because of the technological importance of nanowires and their assemblies, and considerable recent interest in their manipulation and organization. ${ }^{23,25-38}$ Amongst the other possibilities described below, optical binding provides a route to creating ordered, reconfigurable nanowire arrays, potentially deposited onto substrates, with potential applications for photonics, ${ }^{39}$ or nano-electronics. ${ }^{40}$ The technique could be 
(a)

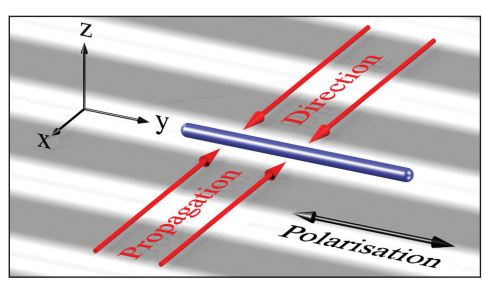

(c)

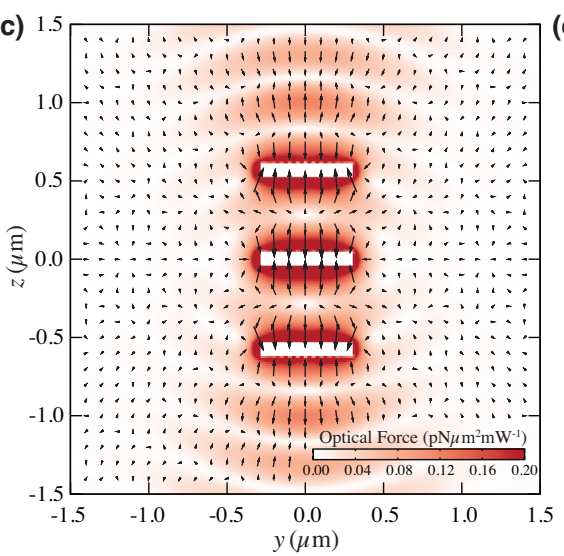

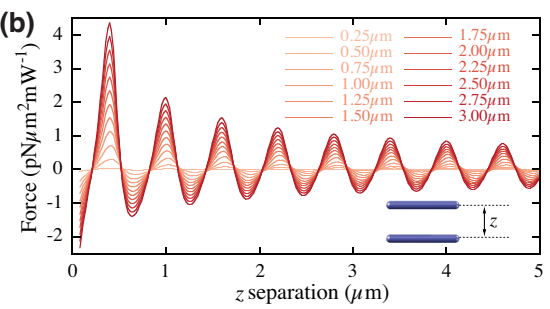

(d)

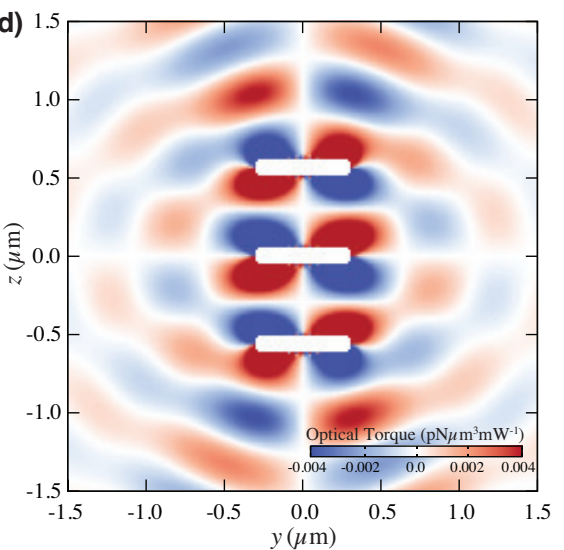

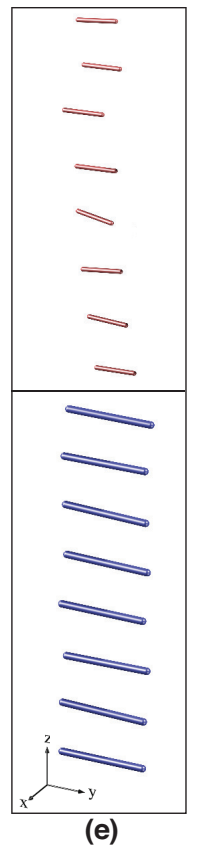

Figure 1: (a) A schematic representation of a nanowire showing the coordinate axes used. The beam propagation direction is parallel to the $x$-axis and the polarisation is along the $y$ axis. The interference fringes are shown projected onto the $x-y$ plane but, in reality, extend parallel to the $z$-axis. (b) The forces acting between pairs of $50 \mathrm{~nm}$ diameter silicon nanowires of different lengths, as a function of separation in the $z$ direction. The nanowires are oriented parallel to the polarization i.e. along the $y$ direction, with their centres on the $z$ axis, both located in the same plane of high intensity created by the counter-propagating waves. (c) Optical forces generated by a ladder of 3 silicon nanowires $(d=50 \mathrm{~nm}, L=300 \mathrm{~nm})$ acting on a fourth nanowire, plotted as a function of its position in the high intensity plane. (d) The corresponding optical torques, acting about the $x$-axis. (e) Snapshots from Brownian dynamics simulations of nanowire ladder structures in the $y-z$ plane with $n=2.5$. Left: $d=50 \mathrm{~nm}, L=0.5 \mu \mathrm{m}$; Right: $d=80 \mathrm{~nm}, L=1.0 \mu \mathrm{m}$. (See Movie M1 in Supplementary Information).

used independently, or in conjunction with other, complementary methods such as flow assisted dielectrophoresis ${ }^{41}$ to produce order at multiple length scales, as required by various integrated nanowire technologies. ${ }^{42}$ We simulate nanowires made from silicon $(n=3.7)$, gallium phosphide $(n=3.2)$ and titanium dioxide $(n=2.5)$. The nanowires are composed of spheres with diameters, $d$, in the range $25.0 \leq d \leq 100.0 \mathrm{~nm}$ and lengths, $L$, varying in increments of $d$, up to a maximum of $5.0 \mu \mathrm{m}$. The geometry of the optical system, comprising counter-propagating plane waves of wavelength $600 \mathrm{~nm}$ in water $(n=1.33)$, is shown in Fig. 1a. Interference produces a sequence of alternating high and low intensity fringes, 
with a periodicity of $\sim 300 \mathrm{~nm}$. The propagation direction is parallel to the $x$-axis so that the fringes form planes perpendicular to $x$. Linearly polarised waves are polarized in the $y$ direction, while for circularly polarized beams, the electric field vector rotates in the $y-z$ plane. Where circularly polarised beams are used, the opposed beams have opposing handedness so that the electric field vectors rotate in the same sense. For fixed configurations, we report optical forces and torques normalized with respect to the optical power, given by the sum of the powers in right and left propagating waves. The absolute power becomes relevant in dynamic simulations, where it determines the relative strengths of systematic and fluctuating forces, influencing hopping rates and the stability of nanowire assemblies. In such situations, we quote the optical powers explicitly. The polarization of the light determines important, general properties of the nanowire dynamics, especially regarding optical torques and rotation. For counter-propagating beams, the available polarization states are remarkably diverse. The examples studied explicitly below are but two instances of the general case, whose qualitative features are described in the Supplementary Information.

The first form of behaviour we describe arises when nanowires are confined to a single bright fringe in linearly polarized, counter-propagating plane waves. In general, nanowires align with the electric polarization vector. Mapping out the optical forces between pairs of nanowires, aligned with the polarization, indicates a significant binding interaction when the nanowires are parallel and separated along the $z$-axis (see Fig. 1b). The optical force between the nanowires oscillates with a period close to the wavelength of the optical field in water, and a sequence of mechanical equilibria are predicted. The binding forces increase with nanowire length and decay approximately inversely with their separation. However, because optical forces are intrinsically non-conservative, such steady state configurations are, strictly, nonequilibrium with non-vanishing fluxes. ${ }^{43,44}$ Asymmetries in the coupling between different degrees of freedom can conspire to push the nanowire ladders out of equilibrium. As a result, non-conservative forces are manifested through continuous or cyclical motions. 

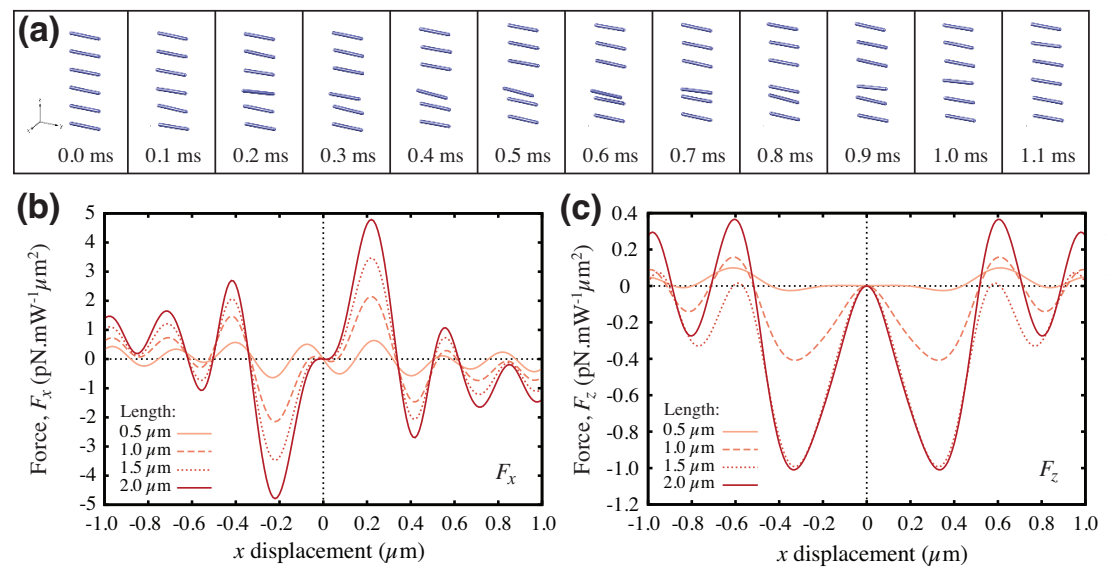

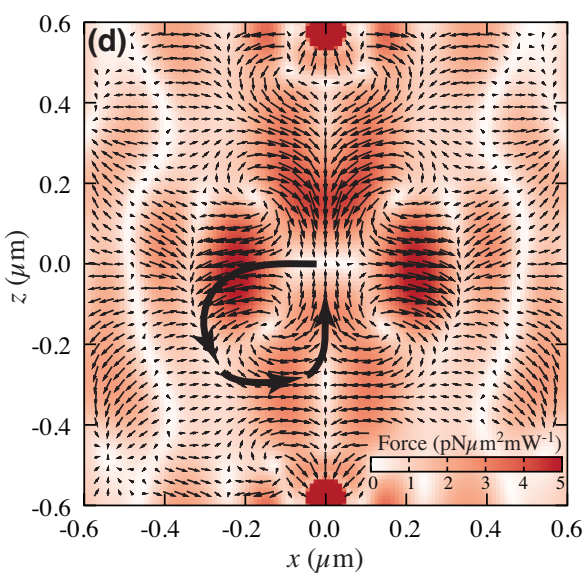

Figure 2: (a) Consecutive frames from a simulation at intervals of $0.1 \mathrm{~ms}$, showing a nanowire move out of a 6-nanowire ladder structure into an adjacent interference fringe, before cycling around and back to its original position (see Movie M2 in Supplementary Information). Motions of this type are frequent and affect all of the nanowires; where multiple excursion coincide, the result may be a permanent loss of the ladder arrangement. In this simulation, $n=2.5, d=100 \mathrm{~nm}$ and $L=1.0 \mu \mathrm{m}$. (b) $x$-component of the optical forces acting on the second rung of a four rung nanowire ladder as it is displaced in the $x$ direction, normal to the plane of the ladder and the interference fringe, for nanowires of varying lengths, $d=50 \mathrm{~nm}$ and $n=3.7$. (c) As (b) but for the $z$-component of the optical force. (d) A vector map showing the forces acting on the second of four nanowires as it is displaced in the $x-z$ plane, for the same system as (b) and (c), but with $L=2.0 \mu \mathrm{m}$. The equilibrium position of the second wire should be at the centre of the plot. The large curved arrows indicate a typical cyclical excursion. 
When several nanowires are placed along the $z$-axis, such that each is at mechanical equilibrium with respect to its neighbour, the binding forces add constructively (Fig. 1c), increasing the magnitude of the binding interaction with subsequently added nanowires. Lateral forces are also apparent, that keep the centres of the bound nanowires to a common axis, resulting in a 1-dimensional ladder-like structure. These accumulating optical interactions also induce optical torques (Fig. 1d), but they are relatively small and the strongest contribution to nanowire orientation is always due to direct interaction with the incident field. The ladder becomes increasingly mechanically stable with respect to in-plane motion, as more rungs (i.e. nanowires) are added. Furthermore, it is found that the optical binding forces increase with increasing nanowire length, thickness and refractive index. However, because optical forces are intrinsically non-conservative, such steady state configurations are, strictly, non-equilibrium with non-vanishing fluxes. ${ }^{43,44}$ Asymmetries in the coupling between different degrees of freedom can conspire to push the nanowire ladders out of equilibrium. As a result, non-conservative forces may be manifested through continuous or cyclical motions, as described below.

A full analysis of the stability of planar bound structures should include out-of-plane motions, and it is found that there are conditions under which nanowire ladders, while conditionally stable with respect to in-plane motion, may become unconditionally unstable with respect to these out-of-plane motions. Brownian dynamics simulations were performed on the nanowire ladders, in order to establish their thermal stability. The ladders were observed to be stable for a range of lengths, thicknesses and refractive indices - example snapshots are shown in Fig. 1e (See Movie M1 in Supplementary Information). Thermal stability was assessed by calculating effective stiffnesses from the variances in coordinates, for various distortions of the ladder structure (see Supplementary Information). Two types of distortion were considered: absolute distortions relating to confinement caused by the incident field, and relative distortions such as shearing between adjacent nanowires and variations in their separation. A general observation is that there is strong confinement of 
the nanowires to the interference fringes and polarisation direction while, within the ladders, shear deformations occur very easily.

The effect of the number and size of nanowires on the stability of the resulting ladders has been explored and two further observations may be made. First, starting with the thinnest nanowires considered (for $n=2.5$ ), there is a general increase in stability as the number of nanowires in the ladder, or their thickness or length is increased. For example, for $d=30 \mathrm{~nm}$ and $L=1.0 \mu \mathrm{m}$, ladders are only thermally stable if they contain more than eight nanowires. Second, for larger thicknesses, the strength of confinement to the bright fringes diminishes with nanowire number such that, e.g., for $d=90$ or $100 \mathrm{~nm}(L=1.0 \mu \mathrm{m})$, ladders are only thermally stable when the number of rungs is less than eight or five respectively. Increasing the nanowire refractive index generally reduces the parameter range for stability (see Supplementary Information Figs. SI.3 and SI.4).

The nature of the ladder instability is identified from the simulation trajectories. The reduction in confinement to a given fringe allows excursions of the nanowires into adjacent fringes. For example, see Fig. 2a (Movie M2 in Supplementary Information): in a cyclic motion, one nanowire is initially displaced in the $x$ direction to an adjacent fringe, then translates by approximately half a rung spacing parallel to $z$, before returning to the original fringe and moving back to its original $z$ position. Increasing the number of nanowires, or the nanowire length, thickness or refractive index, increases the frequency of such hops, resulting in a permanent loss of the ladder structure.

The origin of the instability may be understood by examining the optical forces acting on each nanowire. Two distinct, but related mechanisms operate. The first is illustrated by the plots in Fig. 2b,c. Increasing the length of the wires undermines the restoring force for out-of-plane displacements in the $x$ direction (Fig. 2b). At the same time, displacing wires in the $x$ direction induces an excess force $F_{z}$ in the $z$ direction, arising from the asymmetry of the environment. This combination of forces leads to circulatory motions of the nanowires, as drawn out in Fig. 2d. The repeated cyclic motion ultimately destabilises the ladder. In 
principle, this instability can be counteracted by increasing the optical power, binding the wires more closely to the plane of the ladder.

The second mechanism is more fundamental. The same asymmetries in the wire environments lead to asymmetries in the structure stiffness matrix (see Supplementary Information). This is a fundamental consequence of the non-conservative nature of optical forces, and would not occur for forces derived from a potential. For instance, $d F_{x i} / d x_{j} \neq d F_{x j} / d x_{i}$, for dissimilar nanowires, $i, j$. Closed loops in configuration space appear that are not energetically neutral. In particular, successive displacements of nanowires $i$ and $j$ in the $x$ direction that return the structure to its original configuration will be associated with an overall change in energy. This process gives rise to biased stochastic motion ${ }^{37,38,44}$ and, ultimately, to an instability that cannot be overcome by increasing optical power (see Supplementary Information).

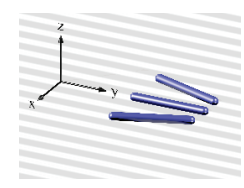

(a)

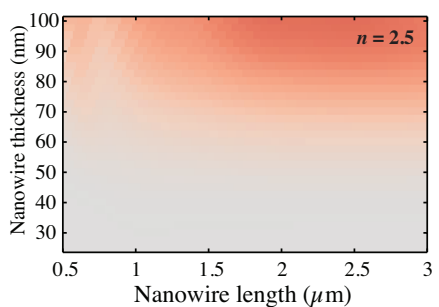

(b)

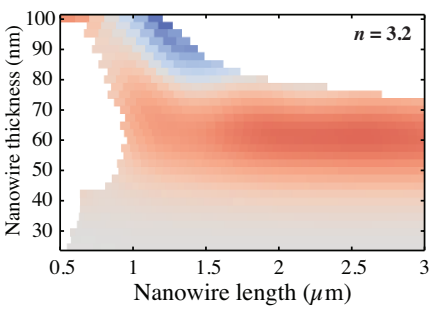

(c)

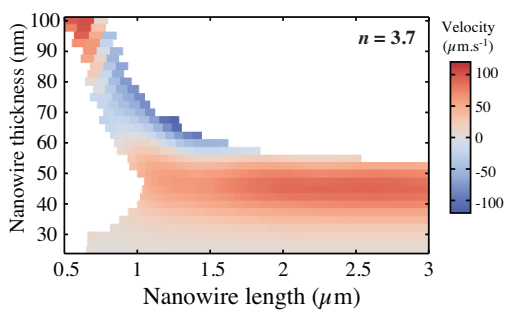

(d)

Figure 3: (a) Schematic of the translating arrow structure. Transverse velocity of arrow formation as a function of length and thickness of the nanowires, for (b) $n=2.5$, (c) $n=3.2$ and (d) $n=3.7$. A beam power of $5 \mathrm{~mW}_{\mu} \mathrm{m}^{-2}$ was assumed. (For example, see Movie M3 in Supplementary Information).

The second form of behaviour we consider arises when nanowires straddle one or more interference fringes. In this case, additional opportunities for symmetry breaking arise. Optical forces arise from the deflection of light by material inhomogeneities. Consequently, the direction of an optical force depends on the shape of the scattering object, with low symmetry particles able to generate force components that are absent in the momentum of the incident field. Notable examples include the stable optical lift of dielectric hemicylinders in linearly polarized plane waves, and the use of conical probes to produce controllable 
transverse forces that function as variable optical springs. ${ }^{45-47}$ In each case, the forces result from asymmetric scattering by the object, which produces compensating optical momentum in the opposite direction.

More recently, optical binding between dissimilar spherical particles has also been shown to produce transverse force components, ${ }^{21,48}$ due to the inequality of action and reaction in a non-conservative system. In the present study it was hoped that transverse forces could be generated in systems of identical nanowires, if stable bound states could be found which lacked a mirror plane. A number of candidate structures were found (see Fig. SI.5); that possessed two inclined nanowires straddling adjacent fringes, and separated by a central wire, in the form of an arrow (see Fig. 3a). The structure is mechanically stable over a wide range of nanowire sizes and refractive indices (see Supplementary Information). However, the structure is not at equilibrium: a pronounced lateral force propels the arrow parallel to the direction of the central wire.

To simulate this system, nanorods were placed into the correct initial configuration. The coherence of the structure is preserved during translation so that the system is in dynamic tension, with viscous forces compensated by optical forces. In effect, this is a primitive, self assembled transducer. The total linear momentum of the incident field is identically zero, but the asymmetry of the nanowire arrangement redirects momentum, generating propulsion. The lateral force (parallel to the $y$ axis) varies with the length, diameter and refractive index of the wires. In a viscous medium, the force is represented by the resultant velocity of the structure, and this is mapped out as a function of nanowire length and thickness for $n=2.5$ in Fig. 3b. Velocities up to several 10's of microns per second in the direction of the arrow, are predicted for a beam power of $5 \mathrm{~mW} \mu \mathrm{m}^{-2}$.

Increasing the refractive index to $n=3.2$ or 3.7 generally increases the predicted velocities for a given nanowire size (see Fig. 3c \& d). However, there are two regions of parameter space (indicated in white) for which the arrow-like structures cease to be stable. Instability sets in for arrows constructed from nanowires which are excessively thick and long (top 
right region of the graph), but also for arrows in which the nanowires are short (left hand region of graph), with a narrow ribbon of stability separating the two areas. The nature of the instability appears to be different for the two regions of parameter space. For shorter nanowires, interactions with the interference fringes dominate over the binding interactions for inclined wires, with the result that the nanowires rotate into a parallel configuration; the loss of asymmetry results in a loss of translation. However, for the longer, thicker nanowires, it is the optical binding interaction which dominates. Inspection of the simulation frames (see Fig. SI.6) indicates that the nanowires move until they are in contact; subsequently the binding forces cause the inclined nanowires to move out of the plane of the arrow and the structure collapses.

Interestingly, the ribbons of parameter space between the two unstable regions coincide with the onset of negative velocities i.e. many of the arrow structures in these regions will travel backwards relative to the direction that the arrow is pointing. Substantial negative velocities are possible for a narrow range of parameter values. Although there is no simple reason, a priori, to expect motion in the direction of the arrow, the change in sign of the velocity is, nevertheless, intriguing and, presumably, arises from a subtle interplay between the binding interactions and the interactions of individual nanowires with the optical fringes, leading to small differences in the net transverse scattering. Similar subtleties in the geometric control of transverse forces have been observed in the design of 3 -d nanotools. ${ }^{47}$

As a final phase of this study, we consider the effect of circular polarization on nanowire binding and non-conservative morion. The total flow of optical momentum associated with pairs of equal, counter-propagating waves, is zero. However, if the constituent waves are circularly polarized with opposing handedness, the light field has a spin density, proportional to $\Im\left(\mathbf{E} \times \mathbf{E}^{\star}\right)$ that can impart torques to the constituent particles. Any non-absorbing cylindrically symmetric particle with its symmetry axis aligned with the propagation direction of a circularly polarised plane wave will not rotate. This is a consequence of the symmetry in the scattered multipoles. ${ }^{49}$ However, when the cylinders are arranged perpendicular to the 


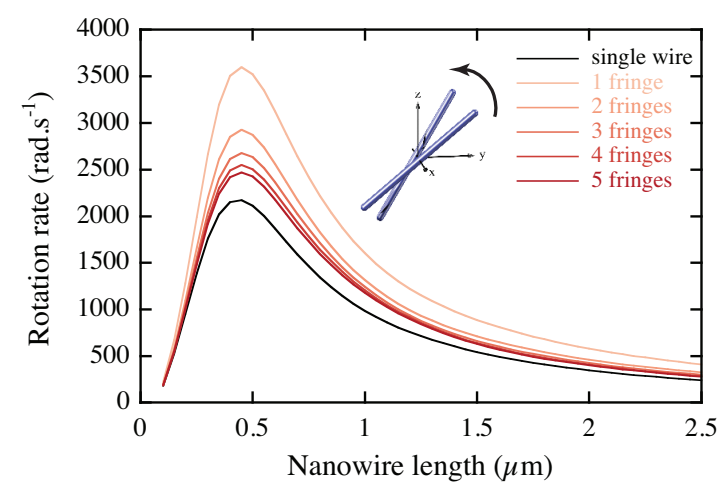

Figure 4: Rotation rates for pairs of nanowires of varying length $(d=50 \mathrm{~nm}, n=3.7)$, separated by varying numbers of fringes, in multiples of $0.3 \mu \mathrm{m}$, compared with the rotation rate for isolated nanowires. A beam power of $5 \mathrm{mW \mu m} \mathrm{m}^{-2}$ is assumed.

propagation direction, the resulting rotational dynamics is extremely rich, showing various forms of synchronization ${ }^{50-52}$ and spin-orbit coupling. ${ }^{53-55}$

Positioning nanorods in different interference fringes in counter-propagating circularly polarised plane waves (Fig. 4 inset) results in synchronous rotation similar to that observed for dielectric spheroids. ${ }^{51,52}$ Optical binding forces act to constrain the centres of the wires to a single axis while spin optical angular momentum causes them to rotate in synchrony, in perfect alignment, at least when thermal fluctuations are neglected. The rotation rates vary with the nanowire lengths, increasing to a maximum at $L \approx 0.5 \mu \mathrm{m}$ (for nanowires with $d=50 \mathrm{~nm}$ ), and then decreasing towards zero (see Fig. 4). Interactions between the nanowires tend to increase the angular velocity, and the rotation rate rises as the separation, determined by the number of intervening fringes, decreases. Adding further nanowires to the structure in adjacent fringes was generally found to disrupt the rotations: when there are more than two nanowires, each is effectively in a different environment, both optically and hydrodynamically, with the result that perfect in-phase synchrony does not occur.

The physical origin of spin-induced optical torque is interesting, and several properties may be discerned by thought experiments. ${ }^{56}$ For example, an infinite rod with its axis normal to the propagation direction of an infinite, circularly polarized plane-wave will not experience a torque, since it is translationally invariant along its axis, and there is no definable centre. 

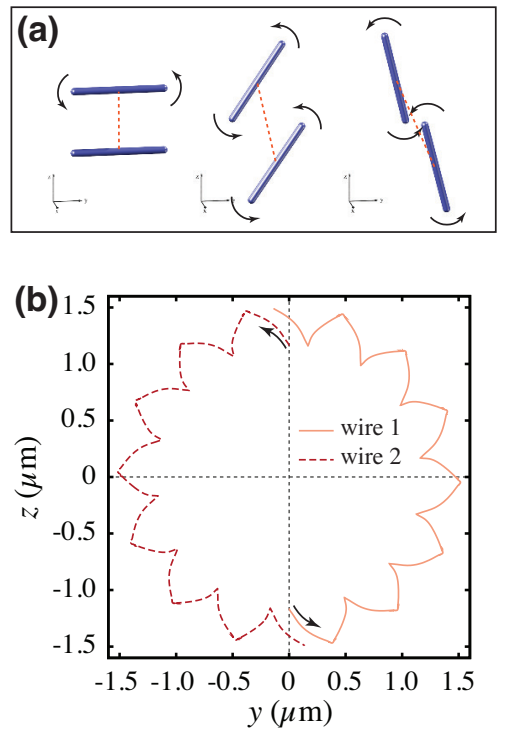
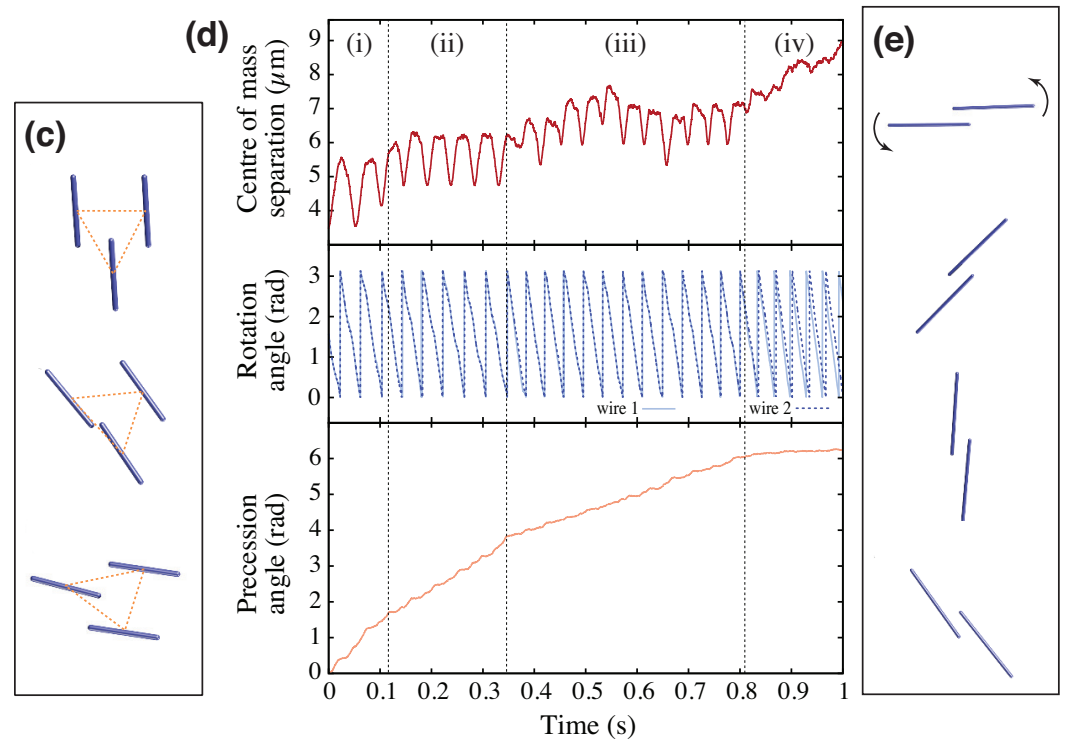

Figure 5: (a) Schematic showing the rotation, and precession, of two nanowires placed adjacently in the same interference fringe of a pair of counter-propagating circularly polarised plane waves. (b) The trajectories of the centres of mass of two nanowires $(d=100 \mathrm{~nm}, L=$ $2.5 \mu \mathrm{m}, n=3.7$, thermal fluctuations suppressed, Movie M4 in Supplementary Information) in the configuration shown in (a). (c) Synchronous rotation of triplets of nanowires (Movie M5 in Supplementary Information). (d) Centre of mass separation, rotation angles and precession angles for a pair of nanowires with $d=100 \mathrm{~nm}, L=5.0 \mu \mathrm{m}$ and $n=3.7$, placed adjacently in the same interference fringe of a set of counter-propagating circularly polarised plane waves with total power $5 m W \mu m^{-2}$, as a function of time (Movie M6 in Supplementary Information). (e) Frames from a simulation of two nanowires initially placed sufficiently close that they could not rotate independently, illustrating the rigid rotation of a bound pair (Movie M7 in Supplementary Information). 
It follows that optically induced torques, routinely observed in experiments, are edge effects, requiring spatial variation perpendicular to the torque axis, either in the field - a finite beam would exert a torque on an infinite rod-or in the particle. Since spin-induced optical torque is an edge effect or, specifically in the present case an end-effect, we can anticipate that the torque acting on a long finite rod in a circularly polarized plane wave will be proportional to $L$ and, since the rotational drag is approximately $\propto L^{3},{ }^{57}$ rotation rates for long wires will decrease as $L^{-2}$, as suggested by Fig. 4 (see S.I. for further discussion).

A further question emerges if we consider cutting the nanowire in half. Will the two halves rotate as if they were still joined, orbiting about their combined centre of mass, or will they rotate separately, about their respective centres of mass? To address this question, we first consider the behaviour of pairs of nanowires, placed side-by-side in a single interference fringe (see Fig. 5a). In the snapshots shown, the two nanowires rotate separately but in synchrony. However, the motion is far from uniform. As the wires rotate, they move apart allowing their ends to pass each other (right hand frame); each time they pass through a parallel configuration (left hand frame) they are attracted and their spacing decreases. A second effect is also seen: the line joining the centres of the two nanowires is gradually rotating i.e. the two nanowires are precessing about their common centre of mass. This is quantified for a pair of nanowires in Fig. 5b.

The rotation and precession rates vary with the lengths of the nanowires and their separation (see Fig. SI.8 and Movie M4 in Supplementary Information). In the absence of thermal fluctuations, the average separation of the nanowire centres is approximately equal to an integer number of wavelengths, and depends on the initial configuration and $L$. This is analogous to the preferred binding separations observed in linearly polarised beams. The spin rotation rates of the nanowires are relatively constant with respect to their average separation, but the precession, or orbital, rates vary over four orders of magnitude for the nanowires studied. In general, the precession rate increases with the length of nanowire and with decreasing separation. Similar effects are found if we consider triplets of nanowires 
(Fig. 5c, Movie M5 in Supplementary Information). These typically form triangular configurations, with an average spacing that is an approximate integer multiple of the wavelength. As previously, the rods rotate and precess about a common centre (Fig. SI.9).

When thermal fluctuations are included, rotation and precession are still observed, although the motion becomes more complex to analyse. For example, Fig. 5d (Movie M6 in Supplementary Information) shows the behaviour of two nanowires with $d=100 \mathrm{~nm}$ and $L=5.0 \mu \mathrm{m}$. The plot may be divided into four distinct regions, as indicated on the figure. Regions (i) - (iii) have increasing average separations (top panel) which correspond to decreasing precession rates (bottom panel). The rotations remain synchronised in these regions. However, in region (iv), the nanowires drift apart as binding is lost. This coincides with a loss both of precession and synchronisation, suggesting that each stem from a common cause.

Finally, we return to the initial question: concerning whether a pair of nanowires might rotate as a single body, held together by optical binding forces. Figure 5e shows frames taken from a simulation in which the initially configuration placed the nanowires too close to rotate without interfering with each other. The two wires bind with their ends overlapping, in a steady-state configuration which is stable provided the system is rotating (Movie M7 in Supplementary Information). The bound pair spins in the optical field, as might be expected. However, from the point of view of the individual nanowires, each effectively acquires orbital angular momentum from the field.

To summarise, these computer simulations show that optical binding between dielectric nanowires leads to many fascinating physical phenomena. Inclusion of the fluid response in the model is crucially important to the observed behaviour since hydrodynamic interactions dictate the kinetic pathway for intrinsically non-equilibrium systems. ${ }^{58,59}$ Furthermore, thermal fluctuations, which scale with the hydrodynamic resistance, determine the stability and coherence of any steady state. The predicted dynamical effects are surprisingly rich. As discussed above, the anisotropy of the interparticle forces favours ladder-like arrangements. 
In common with all other optically bound assemblies, these structures correspond to nonequilibrium steady states, which is quantitatively manifested in the non-symmetric structure stiffness matrix.

In addition, the use of non-spherical particles opens the possibility of lower symmetry, mechanically stable assemblies, such as the arrow shaped triplets that spontaneously translate in a direction perpendicular to the momentum carried by the counter-propagating beams. Although we have focussed attention on one asymmetric configuration, others are possible with yet more complex motion.

We have also demonstrated the coordinated and synchronous rotation of arrays of nanorods. Perhaps most interestingly, a transition has been predicted from effectively rigid rotation of nanowire pairs, to independent rotation, with decreasing binding strength. At intermediate binding strengths, the nanowires both rotate and precess about each other. This behaviour reveals much about the coupling of spin angular momentum to matter. Our simulations show that the application of orbital torques rather than spin torques to discrete parts of the optically bound cluster, is a consequence of the rigidity of the structure, which effectively couples spin to orbital momentum in the mechanical system.

Finally, we comment that the extraordinary richness of the dynamical effects observed has been produced by a very simple reduction in symmetry. Although the particular configurations we have considered are somewhat contrived, experimental systems in which initial conditions are beyond direct control may be expected to produce combinations of the distinct forms of behaviour we have elucidated. Alternatively, additional degrees of experimental control may be introduced by using, for instance, additional optical traps. More generally, the parameter space offered by optically bound nanostructures is vast, and extending the investigation to other classes of particle, will yield yet more complexity. Even a minor change in shape can modify behaviour. For instance, tapered nanowires will experience steady, non-conservative axial forces, ${ }^{47}$ deforming equilibrium configurations, or causing systematic migration of entire assemblies. Although initial calculations suggest these effects are rela- 
tively weak, more general symmetry breaking such as twists or bends will have much more profound consequences. Understanding the driven self-organization of fluctuating assemblies is of fundamental significance. Questions concerning the existence of extremal principles, which might minimize, or maximize, entropy production for example, ${ }^{60}$ or the importance of system history, are fundamental. ${ }^{58}$ Understanding them, through the examination of systems like the one described above, is essential in developing the capacity for the top-down, spontaneous self-organization of nano-machines capable of performing intricate tasks at the nanoscale. ${ }^{61}$

\section{Acknowledgement}

The authors thank the Czech Science Agency (GB14-36681G) for support for S.H.S. and P.Z., CNR and CAS for support for O.M, P.Z. (CNR-16-12) and the University of Bristol Advanced Computing Research Centre (http://acrc.bris.ac.uk/) for provision of computing resources.

\section{Supporting Information Available}

The following files are available free of charge.

- Technical and descriptive details. Detailed movie legends. Optical polarization and torque. Numerical model description. Stability of ladder structures. Stability of arrow structures. Nanowire rotation and precession.

- Movie M1: Brownian dynamics simulations of ladder structures.

- Movie M2: Brownian dynamics simulation of ladder instabilities.

- Movie M3: Brownian dynamics simulation of arrow structures.

- Movie M4: Dynamic simulation of nanowire pair in circularly polarized light.

- Movie M5: Dynamic simulation of nanowire triplet in circularly polarized light. 
- Movie M6: Brownian dynamics simulation of nanowire pair in circularly polarized light.

This material is available free of charge via the Internet at http://pubs.acs.org/.

\section{References}

(1) Mann, S. Nat. Mater. 2009, 8, 781-792.

(2) Yan, Z.; Gray, S. K.; Scherer, N. F. Nat. Commun. 2014, 5.

(3) Antonietti, M.; Ozin, G. A. Chem. Eur. J. 2004, 10, 28-41.

(4) Yu, N.; Capasso, F. Nat. Mater. 2014, 13, 139-150.

(5) Burns, M. M.; Fournier, J.-M.; Golovchenko, J. A. Phys. Rev. Lett. 1989, 63, 1233.

(6) Čižmár, T.; Romero, L. D.; Dholakia, K.; Andrews, D. J. Phys. B 2010, 43, 102001.

(7) Dholakia, K.; Zemánek, P. Rev. Mod. Phys. 2010, 82, 1767.

(8) Karásek, V.; Čižmár, T.; Brzobohatỳ, O.; Zemánek, P.; Garcés-Chávez, V.; Dholakia, K. Phys. Rev. Lett. 2008, 101, 143601.

(9) Brzobohaty, O.; Karasek, V.; Cizmar, T.; Zemanek, P. Appl. Phys. Lett. 2011, 99.

(10) Brzobohaty, O.; Cizmar, T.; Karasek, V.; Siler, M.; Dholakia, K.; Zemanek, P. Opt. Express 2010, 18, 25389-25402.

(11) Brzobohaty, O.; Karasek, V.; Siler, M.; Chvatal, L.; Cizmar, T.; Zemanek, P. Nat. Photon. 2013, 7, 123-127.

(12) Han, X.; Luo, H.; Xiao, G.; Jones, P. H. Opt. Lett. 2016, 41, 4935-4938.

(13) Ng, J.; Lin, Z.; Chan, C.; Sheng, P. Phys. Rev. B 2005, 72. 
(14) Maragò, O. M.; Jones, P. H.; Gucciardi, P. G.; Volpe, G.; Ferrari, A. C. Nat. Nanotechnol. 2013, 8, 807-819.

(15) Yan, Z.; Shah, R. A.; Chado, G.; Gray, S. K.; Pelton, M.; Scherer, N. F. ACS nano 2013, \%, 1790-1802.

(16) Bradshaw, D. S.; Andrews, D. L. Phys. Rev. A 2005, 72, 033816.

(17) Forbes, K. A.; Andrews, D. L. Phys. Rev. A 2015, 91, 053824.

(18) Grzegorczyk, T. M.; Kemp, B. A.; Kong, J. A. JOURNAL OF THE OPTICAL SOCIETY OF AMERICA A-OPTICS IMAGE SCIENCE AND VISION 2006, 23, 23242330 .

(19) Rodriguez, J.; Romero, L. C. D.; Andrews, D. L. Journal of Nanophotonics 2007, 1, 019503-019503.

(20) Bao, Y.; Yan, Z.; Scherer, N. F. The Journal of Physical Chemistry C 2014, 118, 19315-19321.

(21) Sukhov, S.; Shalin, A.; Haefner, D.; Dogariu, A. Opt. Express 2015, 23, 247-252.

(22) Ermak, D. L.; McCammon, J. The Journal of chemical physics 1978, 69, 1352-1360.

(23) Andrews, D. L.; Bradshaw, D. S. Opt. Lett. 2005, 30, 783-785.

(24) Ekeroth, R. M. A. Journal of Optics 2016, 18, 085003.

(25) Pauzauskie, P. J.; Radenovic, A.; Trepagnier, E.; Shroff, H.; Yang, P.; Liphardt, J. Nat. Mater. 2006, 5, 97-101.

(26) Agarwal, R.; Ladavac, K.; Roichman, Y.; Yu, G.; Lieber, C.; Grier, D. Opt. Express 2005, 13, 8906-8912. 
(27) Marago, O.; Jones, P.; Bonaccorso, F.; Scardaci, V.; Gucciardi, P.; Rozhin, A.; Ferrari, A. Nano Lett. 2008, 8, 3211-3216.

(28) Irrera, A.; Artoni, P.; Saija, R.; Gucciardi, P. G.; Iatì, M. A.; Borghese, F.; Denti, P.; Iacona, F.; Priolo, F.; Marago, O. M. Nano Lett. 2011, 11, 4879-4884.

(29) Nakayama, Y.; Pauzauskie, P. J.; Radenovic, A.; Onorato, R. M.; Saykally, R. J.; Liphardt, J.; Yang, P. Nature 2007, 447, 1098-1101.

(30) Jamshidi, A.; Pauzauskie, P. J.; Schuck, P. J.; Ohta, A. T.; Chiou, P.-Y.; Chou, J.; Yang, P.; Wu, M. C. Nat. Photon. 2008, 2, 86-89.

(31) Pauzauskie, P. J.; Jamshidi, A.; Valley, J. K.; Satcher Jr, J. H.; Wu, M. C. Appl. Phys. Lett. 2009, 95, 113104.

(32) Reece, P. J.; Paiman, S.; Abdul-Nabi, O.; Gao, Q.; Gal, M.; Tan, H. H.; Jagadish, C. Appl. Phys. Lett. 2009, 95, 101109.

(33) Reece, P. J.; Toe, W. J.; Wang, F.; Paiman, S.; Gao, Q.; Tan, H. H.; Jagadish, C. Nano Lett. 2011, 11, 2375-2381.

(34) Wang, F.; Reece, P. J.; Paiman, S.; Gao, Q.; Tan, H. H.; Jagadish, C. Nano Lett. 2011, 11, 4149-4153.

(35) Wang, F.; Toe, W. J.; Lee, W. M.; McGloin, D.; Gao, Q.; Tan, H. H.; Jagadish, C.; Reece, P. J. Nano Lett. 2013, 13, 1185-1191.

(36) Brzobohaty, O.; Siler, M.; Trojek, J.; Chvatal, L.; Karasek, V.; Patak, A.; Pokorna, Z.; Mika, F.; Zemanek, P. SCIENTIFIC REPORTS 2015, 5.

(37) Irrera, A.; Magazzu, A.; Artoni, P.; Simpson, S. H.; Hanna, S.; Jones, P. H.; Priolo, F.; Gucciardi, P. G.; Marago, O. M. Nano Lett. 2016, 16, 4181-4188. 
(38) Toe, W. J.; Ortega-Piwonka, I.; Angstmann, C. N.; Gao, Q.; Tan, H. H.; Jagadish, C.; Henry, B. I.; Reece, P. J. Phys. Rev. E 2016, 93.

(39) Noori, Y. J.; Cao, Y.; Roberts, J.; Woodhead, C.; Bernardo-Gavito, R.; Tovee, P.; Young, R. J. ACS Photonics 2016, 3, 2515-2520.

(40) Yan, H.; Choe, H. S.; Nam, S.; Hu, Y.; Das, S.; Klemic, J. F.; Ellenbogen, J. C.; Lieber, C. M. Nature 2011, 470, 240-244.

(41) Oh, K.; Chung, J.-H.; Riley, J. J.; Liu, Y.; Liu, W. K. Langmuir 2007, 23, 11932-11940.

(42) Whang, D.; Jin, S.; Wu, Y.; Lieber, C. M. Nano Lett. 2003, 3, 1255-1259.

(43) Roichman, Y.; Sun, B.; Stolarski, A.; Grier, D. G. Phys. Rev. Lett. 2008, 101, 128301.

(44) Simpson, S. H.; Hanna, S. Phys. Rev. E 2010, 82, 031141.

(45) Swartzlander Jr, G. A.; Peterson, T. J.; Artusio-Glimpse, A. B.; Raisanen, A. D. Nat. Photon. 2011, 5, 48-51.

(46) Simpson, S. H.; Hanna, S.; Peterson, T. J.; Swartzlander, G. A. Opt. Lett. 2012, 37, $4038-4040$.

(47) Phillips, D.; Padgett, M.; Hanna, S.; Ho, Y.-L.; Carberry, D.; Miles, M.; Simpson, S. Nat. Photon. 2014, 8, 400-405.

(48) Chvatal, L.; Brzobohaty, O.; Zemanek, P. Opt. Rev. 2015, 22, 157-161, 1st Optical Manipulation Conference (0MC), Yokohama, JAPAN, APR 22-25, 2014.

(49) Marston, P.; Crichton, J. Phys. Rev. A 1984, 30, 2508-2516.

(50) Kotar, J.; Debono, L.; Bruot, N.; Box, S.; Phillips, D.; Simpson, S.; Hanna, S.; Cicuta, P. Phys. Rev. Lett. 2013, 111. 
(51) Brzobohatỳ, O.; Arzola, A. V.; Šiler, M.; Chvátal, L.; Jákl, P.; Simpson, S.; Zemánek, P. Opt. Express 2015, 23, 7273-7287.

(52) Simpson, S. H.; Chvátal, L.; Zemánek, P. Opt. Express 2015, 23, 7273-7287.

(53) Antognozzi, M.; Bermingham, C. R.; Harniman, R. L.; Simpson, S.; Senior, J.; Hayward, R.; Hoerber, H.; Dennis, M. R.; Bekshaev, A. Y.; Bliokh, K. Y.; Nori, F. Nat. Phys. 2016, 12, 731-735.

(54) Sukhov, S.; Kajorndejnukul, V.; Naraghi, R. R.; Dogariu, A. Nat. Photon. 2015, 9, 809-812.

(55) Hayat, A.; Mueller, J. B.; Capasso, F. Proc. Natl. Acad. Sci. U.S.A. 2015, 112, 1319013194.

(56) Allen, L.; Padgett, M. Am. J. Phys. 2002, 70, 567-568.

(57) Kim, S.; Karrila, S. J. Microhydrodynamics: principles and selected applications; Dover Publications, 2013.

(58) England, J. L. Nat. Nanotech. 2015, 10, 919-923.

(59) Royall, C. P.; Eggers, J.; Furukawa, A.; Tanaka, H. Phys. Rev. Lett. 2015, 114, 258302.

(60) Hubler, A.; Belkin, A.; Bezryadin, A. Complexity 2015, 20, 8-11.

(61) Ozin, G. A.; Manners, I.; Fournier-Bidoz, S.; Arsenault, A. Adv. Mater. 2005, 17, 3011-3018. 


\section{Graphical TOC Entry}

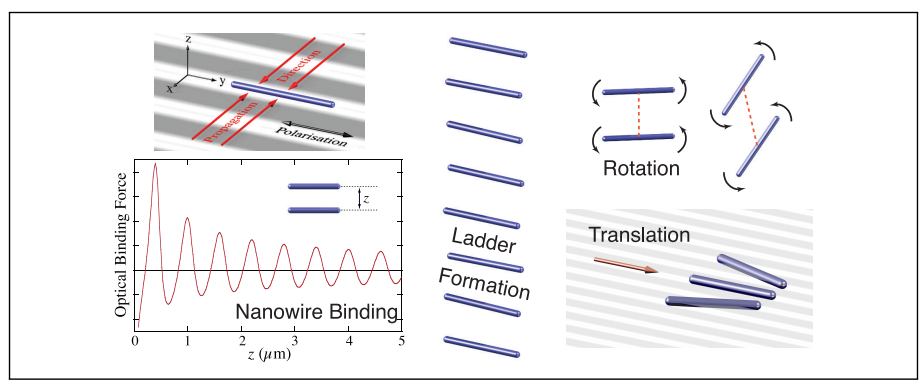

